OPEN ACCESS

Edited by:

Ekaterina Shelest

Leibniz Institute for Natural Product Research and Infection Biology, Hans-Knoell Institute, Germany

Reviewed by:

Pavel Loskot,

Swansea University, UK Tom Thorne,

University of Edinburgh, UK

${ }^{*}$ Correspondence: Smadar Ben-Tabou de-Leon sben-tab@univ.haifa.ac.ll

Specialty section: This article was submitted to Bioinformatics and Computational

Biology,

a section of the journa

Frontiers in Genetics

Received: 02 December 2015 Accepted: 28 January 2016 Published: 15 February 2016

Citation: Ben-Tabou de-Leon S (2016) Robustness and Accuracy in Sea Urchin Developmental Gene

Regulatory Networks.

Front. Genet. 7:16

doi: 10.3389/fgene.2016.00016

\section{Robustness and Accuracy in Sea Urchin Developmental Gene Regulatory Networks}

\author{
Smadar Ben-Tabou de-Leon * \\ The Department of Marine Biology, The University of Haifa, Haifa, Israel
}

Developmental gene regulatory networks robustly control the timely activation of regulatory and differentiation genes. The structure of these networks underlies their capacity to buffer intrinsic and extrinsic noise and maintain embryonic morphology. Here I illustrate how the use of specific architectures by the sea urchin developmental regulatory networks enables the robust control of cell fate decisions. The Wnt- $\beta$ catenin signaling pathway patterns the primary embryonic axis while the BMP signaling pathway patterns the secondary embryonic axis in the sea urchin embryo and across bilateria. Interestingly, in the sea urchin in both cases, the signaling pathway that defines the axis controls directly the expression of a set of downstream regulatory genes. I propose that this direct activation of a set of regulatory genes enables a uniform regulatory response and a clear cut cell fate decision in the endoderm and in the dorsal ectoderm. The specification of the mesodermal pigment cell lineage is activated by Delta signaling that initiates a triple positive feedback loop that locks down the pigment specification state. I propose that the use of compound positive feedback circuitry provides the endodermal cells enough time to turn off mesodermal genes and ensures correct mesoderm vs. endoderm fate decision. Thus, I argue that understanding the control properties of repeatedly used regulatory architectures illuminates their role in embryogenesis and provides possible explanations to their resistance to evolutionary change.

\begin{abstract}
Keywords: developmental gene regulatory network, development and evolution, compound network motifs, sea urchins, Wnt signaling pathway, BMP signaling, Delta-Notch signaling
\end{abstract}

\section{INTRODUCTION}

Robustness, the perseverance of phenotype through genetic and environmental changes (de Visser et al., 2003), is a prominent property of embryo development. Thus, embryos can maintain their morphologies through a wide range of temperatures and pH (Runcie et al., 2012; Pespeni et al., 2013; Kuntz and Eisen, 2014) and within substantial genetic variation (Garfield et al., 2013). This robustness of the developmental program relays on various levels of molecular control, among them, transcription factor binding to the DNA, enhancer structure and the architecture of developmental gene regulatory networks (reviewed in de Visser et al., 2003; Kitano, 2007; Payne and Wagner, 2015). Here I describe the repeated use of specific network architectures in the sea urchin developmental gene regulatory networks, and illustrate how they contribute to robust cell fate decision.

The current model of the sea urchin developmental regulatory networks encompasses all the embryonic territories up to gastrulation and is one of the most elaborate of its kind 
(Saudemont et al., 2010; Peter and Davidson, 2011; Materna and Davidson, 2012; Ben-Tabou de-Leon et al., 2013). A major strength of this network model is the extensive cis-regulatory analyses conducted for many nodes (e.g., Nam et al., 2007; BenTabou de Leon and Davidson, 2010; Ransick and Davidson, 2012). Thus, the direct connectivity of this network is highly reliable and can provide a systems level view of how network architecture contributes to the precise control of embryonic axes formation and germ layer specification.

Within the sea urchin regulatory network, specific network architectures are repeatedly used to control various patterning events at different embryonic territories (Ben-Tabou deLeon and Davidson, 2006; Peter and Davidson, 2009). These network architectures are composed of multiple interconnected common network motifs: switches, feedforward and feedback loops (Ben-Tabou de-Leon and Davidson, 2006; Peter and Davidson, 2009). The concept of "common network motifs" originated more than a decade ago by Alon and colleagues that identified typical three-node network circuitries overrepresented in bacterial transcriptional regulatory networks (Shen-Orr et al., 2002). Since then, similar and other network motifs were identified in other biological systems and their intensive study illuminates the relationship between motif structure and its control function (Hornung and Barkai, 2008; Shoval and Alon, 2010). Here I illustrate how compound interconnected network motifs are used by the sea urchin developmental gene regulatory networks and propose that their control properties are utilized to ensure robustness and accuracy of cell fate decisions.

\section{WNT- $\beta$ CATENIN REGULATION OF PRIMARY AXIS FORMATION AND ENDODERM SPECIFICATION}

Extensive research had shown the extreme conservation of the role of the Wnt- $\beta$ catenin signaling pathway in primary axis formation and endoderm specification across metazoan (Petersen and Reddien, 2009). The model of the sea urchin developmental regulatory networks reveal how Wnt- $\beta$ catenin spatial information is transformed into specific cell fate decisions. The primary axis in the sea urchin embryo, the animal-vegetal axis, is initiated by nuclear localization of $\beta$ catenin in all the cells of the vegetal half of the embryo [Figure 1A, endomesodermal lineages, B, $\beta$ catenin nuclearization pattern (Logan et al., 1999)]. When $\beta$ catenin enters the nucleus it forms an activating complex with the transcription factor Tcf that otherwise forms a repressor complex with Groucho. The $\beta$ catenin-Tcf switch initiates the specification of both mesoderm and endoderm in the vegetal half of the sea urchin embryo (Figures 1A-E).

$\beta$ catenin-Tcf switch directly activates the expression of a set of endodermal regulatory genes, hox 11/13, blimp1, foxa, and bra, in a staggered manner [Figure 1C (Cui et al., 2014)]. That is, the expression of each of these gene is turned on at a different time, but their spatial expression overlap, at least at the earlier stages of their expression (Minokawa et al., 2005; Livi and Davidson, 2006; Peter and Davidson, 2010, 2011). Each of these genes has functional Tcf sites in its enhancers, indicating direct control of Wnt signaling through $\beta$ catenin /Groucho-Tcf switch (Figure 1F, Minokawa et al., 2005; Smith et al., 2007, 2008; Ben-Tabou de Leon and Davidson, 2010).

At Mesenchyme blastula stage, $\beta$ catenin clears from the mesodermal nuclei, first from the skeletogenic lineage and then from the non-skeletogenic mesoderm [Figure 1B (Logan et al., 1999)]. When $\beta$ catenin is cleared from the mesodermal nuclei the Tcf sites on the enhancers of the endodermal genes control their clearance from the mesoderm territories through Tcf-Groucho mediated repression (Ben-Tabou de Leon and Davidson, 2010) and thus regulate the endoderm-mesoderm cell fate decision (Figure 1F, Peter and Davidson, 2011). Apparently, ßcateninTcf acts as a permissive switch and restricts the expression of these genes spatially, while their differential activation time is defined by their specific activators (Figure 1F). I suggest that this mode of regulatory circuitry decouples the spatial from the temporal regulation and promotes a uniform spatial response of all the endodermal genes. Thus, $\beta$ catenin-Tcf/Groucho-Tcf switch ensures that the endodermal genes will be cleared from the mesodermal domain at the right developmental stage and guarantees a clear-cut cell fate decision.

\section{DELTA-NOTCH ACTIVATION OF A TRIPLE POSITIVE FEEDBACK CIRCUIT AND MESODERM CELL FATE SPECIFICATION}

The Delta-Notch signaling pathway is highly conserved in metazoan and controls glial vs. neural differentiation (Gaiano and Fishell, 2002). Early in sea urchin embryogenesis, the gene that encodes the ligand Delta is activated indirectly by the $\beta$ catenin-Tcf input in the skeletogenic mesoderm (Figure 1D, Oliveri et al., 2008). The reception of Delta in the neighboring tier of cells, Veg2, activates the gene that encodes the transcription factor glial cells missing [GCM, Figures 1E,G (Ransick and Davidson, 2006; Croce and McClay, 2010)]. GCM then establishes a triple positive feedback loop by directly activating the expression of the transcription factor GataE, that activates the expression of the transcription factor Six $1 / 2$, that feeds back to activate GCM expression (Figures 1E,G, Ransick and Davidson, 2012). GCM-GataE-Six1/2 triple positive feedback loop maintains the expression of these three genes in the pigment cell lineage after Delta signal stops being received in these cells (20 hpf in S. purpuratus, Figures 1D,E,G).

The tier of cells where GCM is first activated, Veg2, give rise to both endoderm and non-skeletogenic mesoderm lineages (Figure 1A, 12 hpf). When Veg2 cells divide, only the future pigment cells remain in direct contact with the Delta secreting SM cells, while the future endodermal cells lose this contact and therefore lose the Delta signal (Figure 1A, 15 hpf). Hence, in the endodermal cells the Delta signal is not received long enough to establish the triple positive feedback loop so GCM expression turns off there (Figure 1E, 15 and 20 hpf, Ransick and Davidson, 2006, 2012; Croce and McClay, 2010). The transient Delta signal is practically filtered in the endodermal cells by the mesodermal positive feedback loop to allow correct endodermal fate decision. 


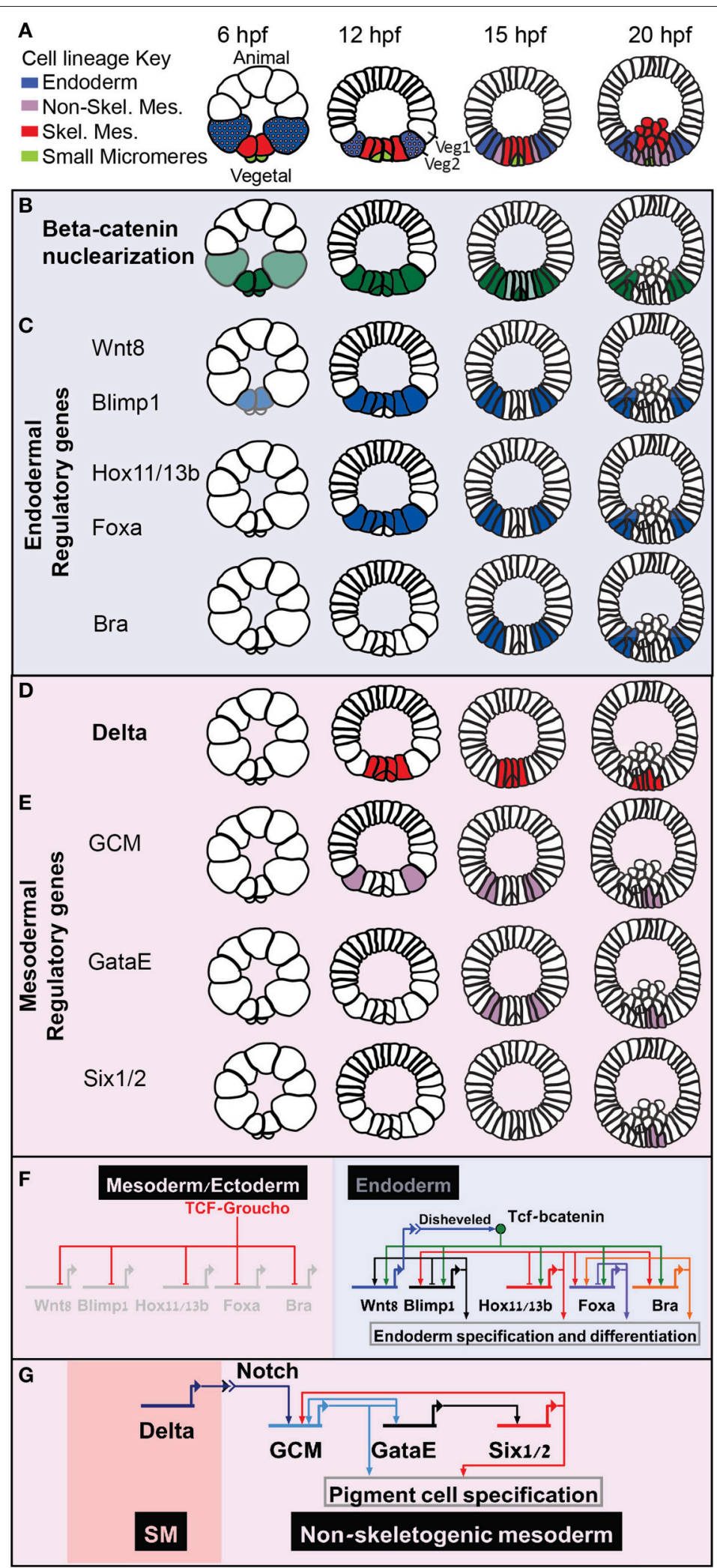

FIGURE 1 | Sea urchin embryonic development and endoderm specification. Developmental time is described in hours post fertilization according the developmental rate of the purple sea urchin, S. purpuratus. (A) Sea urchin endomesoderm cell lineage diagram. Color key is described in the figure. (B) $\beta$ catenin nuclearization pattern, dark green indicates high concentration, light green low. (C) Spatio-temporal expression profiles of endodermal control genes. (D) Partial 
FIGURE 1 | Continued

endodermal GRN model depicting Tcf/Bcatenin-Tcf/Groucho switch and regulatory interactions within the endodermal genes. (E) Spatio-temporal expression of the Delta ligand. (F) spatio-temporal expression of non-skeletogenic mesodermal genes. (G) GRN model of the triple positive feedback loop that Delta reception activates in the non-skeletogenic cells.

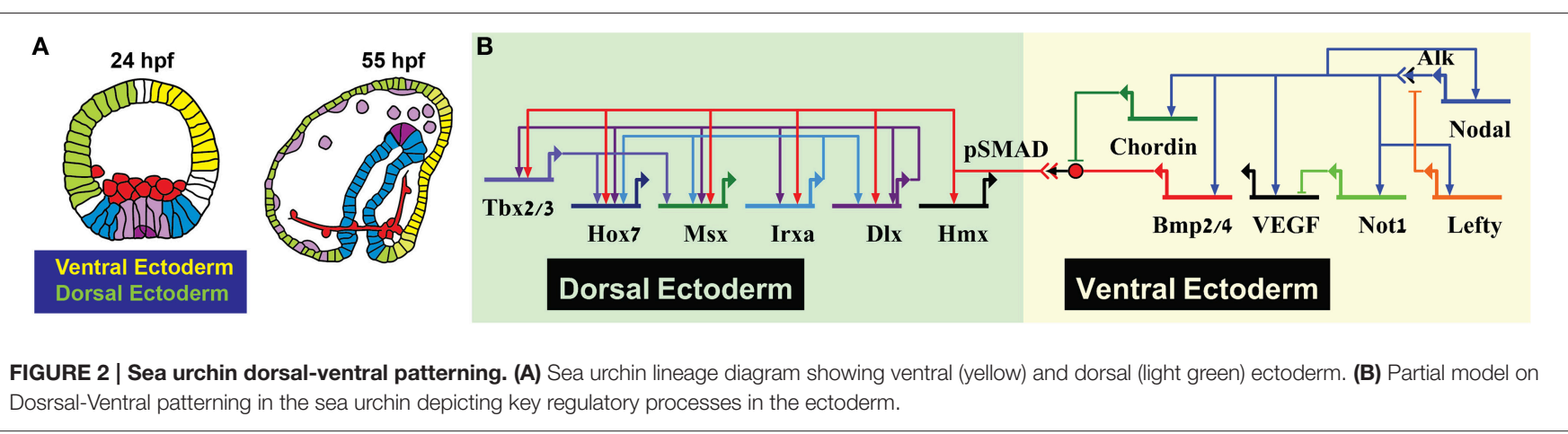

Previous theoretical studies of three component circuits show that feedback circuitry is more efficient than other architectures in buffering noise in the inducing signal while keeping high responsivity to the level of the signal (Hornung and Barkai, 2008). According to these studies, noise reduction in positive feedback circuits results from effectively slowing the response dynamics and allowing for better averaging of the induction signal over time. Additionally, mathematical modeling of the kinetics of positive feedback loops shows that compound positive feedback circuitry is less responsive than single positive feedback loop to low levels of activating signals (Ben-Tabou de-Leon, 2010). These studies suggests that compound positive feedback circuitry filters better low and transient signals compared to single positive feedback loops and thus are a more reliable mechanism for regulatory state lock down. This could be the reason for the common use of compound positive feedback circuits by developmental networks instead of single gene positive feedback loop.

\section{TGFB PATHWAYS CONTROL OF SECONDARY AXIS AND ECTODERM SPECIFICATION}

The gene regulatory networks that pattern the secondary embryonic axis, the dorsal-ventral axis of the sea urchin embryo, use similar circuit architectures to those discussed above. Nodal signaling directly activates the ventral ectoderm regulatory genes that then interact with each other to form subdomains within the ventral ectoderm [Figure 2 (Saudemont et al., 2010; Li et al., 2014)]. Two of Nodal targets at the ventral ectoderm are the ligand BMP2/4 and its inhibitor Chordin. Chordin inhibits BMP reception at the ventral side so the mediator of BMP signaling, the transcription factor SMAD1/5/8, is phosphorylated and activates transcription only in the dorsal side of the embryo (Figure 2B, Saudemont et al., 2010; Ben-Tabou de-Leon et al., 2013). BMP operates in a feed-forward structure, directly activating the expression of dorsal transcription factors that then regulate one another forming compound positive feedback loop (Figure 2B, Ben-Tabou de-Leon et al., 2013). Thus, BMP provides a temporal cue that uniformly boosts the expression of the aboral transcription factors at the exact time when the first genes that specify the neighboring territory, the ciliated band, are turning on (Ben-Tabou de-Leon et al., 2013).

\section{CONCLUSIONS: PRECISE AND HIGHLY CONSERVED CONTROL OF EXPRESSION DYNAMICS}

As we gain more information on the structure and function of gene regulatory networks we can start asking why are specific architectures used more than others and why are they so deeply conserved? A recent paper revealed remarkable conservation of regulatory gene expression dynamics between two sea urchin species after 40 million years of independent evolution (Gildor and Ben-Tabou de-Leon, 2015). The use of direct activation by signaling pathways and compound positive feedback circuitry described above could underlie this strong conservation of expression dynamics and the observed robustness within genotypic variance and different environmental conditions.

Direct activation by a signaling pathway might be a general strategy used by developmental gene regulatory networks to guarantees a uniform timely response of a set of key regulatory genes. This strategy could also explain the deep conservation of the role of Wnt and BMP pathways in primary embryonic axes specification. If the activation of the downstream gene regulatory network was in a cascade of regulatory interactions, there were only a few regulatory changes required to replace Wnt or BMP with alternative signaling input. It is much less likely to replace Wnt or BMP signaling when they activate the entire set of genes that define the endoderm or dorsal ectoderm specification, respectively. Thus, the direct activation of large sets of regulatory genes by signaling pathways might be important for clear cut cell fate decision on one hand, and on the other hand imposes 
a strong constraint on the use of these signaling pathways in developing embryos.

Similar argument could explain the extreme conservation of well-studied compound positive feedback circuits. Specifically, the compound positive feedback circuit that controls the lock down of endoderm cell fate specification was conserved across 500 years of echinoderm evolution (Hinman et al., 2003); the compound positive feedback circuit that controls heart development is conserved between human and fly (Olson, 2006). It seems that any regulatory change within these critical control circuits must have reduced the circuit precision and therefore had been selected against. Thus, understanding the control properties of repeatedly used regulatory architectures illuminates

\section{REFERENCES}

Ben-Tabou de-Leon, S. (2010). Perturbation analysis analyzed - mathematical modeling of intact and perturbed gene regulatory circuits for animal development. Dev. Biol. 344, 1110-1118. doi: 10.1016/j.ydbio.2010.06.020

Ben-Tabou de Leon, S., and Davidson, E. H. (2010). Information processing at the foxa node of the sea urchin endomesoderm specification network. Proc. Natl. Acad. Sci. U.S.A. 107, 10103-10108. doi: 10.1073/pnas.1004824107

Ben-Tabou de-Leon, S., and Davidson, E. H. (2006). Deciphering the underlying mechanism of specification and differentiation: the sea urchin gene regulatory network. Sci. STKE 2006:pe47. doi: 10.1126/stke.3612006pe47

Ben-Tabou de-Leon, S., Su, Y. H., Lin, K. T., Li, E., and Davidson, E. H. (2013). Gene regulatory control in the sea urchin aboral ectoderm: spatial initiation, signaling inputs, and cell fate lockdown. Dev. Biol. 374, 245-254. doi: 10.1016/j.ydbio.2012.11.013

Croce, J. C., and McClay, D. R. (2010). Dynamics of Delta/Notch signaling on endomesoderm segregation in the sea urchin embryo. Development 137, 83-91. doi: $10.1242 / \mathrm{dev} .044149$

Cui, M., Siriwon, N., Li, E., Davidson, E. H., and Peter, I. S. (2014). Specific functions of the Wnt signaling system in gene regulatory networks throughout the early sea urchin embryo. Proc. Natl. Acad. Sci. U.S.A. 111, E5029-E5038. doi: 10.1073/pnas.1419141111

de Visser, J. A., Hermisson, J., Wagner, G. P., Ancel Meyers, L., Bagheri-Chaichian, H., Blanchard, J. L., et al. (2003). Perspective: evolution and detection of genetic robustness. Evolution 57, 1959-1972. doi: 10.1111/j.0014-3820.2003.tb00377.x

Gaiano, N., and Fishell, G. (2002). The role of notch in promoting glial and neural stem cell fates. Annu. Rev. Neurosci. 25, 471-490. doi: 10.1146/annurev.neuro.25.030702.130823

Garfield, D. A., Runcie, D. E., Babbitt, C. C., Haygood, R., Nielsen, W. J., and Wray, G. A. (2013). The impact of gene expression variation on the robustness and evolvability of a developmental gene regulatory network. PLoS Biol. 11:e1001696. doi: 10.1371/journal.pbio.1001696

Gildor, T., and Ben-Tabou de-Leon, S. (2015). Comparative study of regulatory circuits in two sea urchin species reveals tight control of timing and high conservation of expression dynamics. PLoS Genet. 11:e1005435. doi: 10.1371/journal.pgen.1005435

Hinman, V. F., Nguyen, A. T., Cameron, R. A., and Davidson, E. H. (2003). Developmental gene regulatory network architecture across 500 million years of echinoderm evolution. Proc. Natl. Acad. Sci. U.S.A. 100, 13356-13361. doi: $10.1073 /$ pnas. 2235868100

Hornung, G., and Barkai, N. (2008). Noise propagation and signaling sensitivity in biological networks: a role for positive feedback. PLoS Comput. Biol. 4:e8. doi: 10.1371/journal.pcbi.0040008

Kitano, H. (2007). Towards a theory of biological robustness. Mol. Syst. Biol. 3:137. doi: $10.1038 / \mathrm{msb} 4100179$

Kuntz, S. G., and Eisen, M. B. (2014). Drosophila embryogenesis scales uniformly across temperature in developmentally diverse species. PLoS Genet. 10:e1004293. doi: 10.1371/journal.pgen.1004293

Li, E., Cui, M., Peter, I. S., and Davidson, E. H. (2014). Encoding regulatory state boundaries in the pregastrular oral ectoderm of the sea urchin their function in developing embryos and provides possible explanation to their resistance to evolutionary change.

\section{AUTHOR CONTRIBUTIONS}

The author confirms being the sole contributor of this work and approved it for publication.

\section{FUNDING}

This work was supported by the Marie Curie Carrier Integration Grant FP7-PEOPLE-2012-CIG, grant number 321758.

embryo. Proc. Natl. Acad. Sci. U.S.A. 111, E906-E913. doi: 10.1073/pnas.1323 105111

Livi, C. B., and Davidson, E. H. (2006). Expression and function of blimp1/krox, an alternatively transcribed regulatory gene of the sea urchin endomesoderm network. Dev. Biol. 293, 513-525. doi: 10.1016/j.ydbio.2006.02.021

Logan, C. Y., Miller, J. R., Ferkowicz, M. J., and McClay, D. R. (1999). Nuclear beta-catenin is required to specify vegetal cell fates in the sea urchin embryo. Development 126, 345-357.

Materna, S. C., and Davidson, E. H. (2012). A comprehensive analysis of Delta signaling in pre-gastrular sea urchin embryos. Dev. Biol. 364, 77-87. doi: 10.1016/j.ydbio.2012.01.017

Minokawa, T., Wikramanayake, A. H., and Davidson, E. H. (2005). cis-Regulatory inputs of the wnt8 gene in the sea urchin endomesoderm network. Dev. Biol. 288, 545-558. doi: 10.1016/j.ydbio.2005.09.047

Nam, J., Su, Y. H., Lee, P. Y., Robertson, A. J., Coffman, J. A., and Davidson, E. H. (2007). Cis-regulatory control of the nodal gene, initiator of the sea urchin oral ectoderm gene network. Dev. Biol. 306, 860-869. doi: 10.1016/j.ydbio.2007.03.033

Oliveri, P., Tu, Q., and Davidson, E. H. (2008). Global regulatory logic for specification of an embryonic cell lineage. Proc. Natl. Acad. Sci. U.S.A. 105, 5955-5962. doi: 10.1073/pnas.0711220105

Olson, E. N. (2006). Gene regulatory networks in the evolution and development of the heart. Science 313, 1922-1927. doi: 10.1126/science.1132292

Payne, J. L., and Wagner, A. (2015). Mechanisms of mutational robustness in transcriptional regulation. Front. Genet. 6:322. doi: 10.3389/fgene.2015.00322

Pespeni, M. H., Chan, F., Menge, B. A., and Palumbi, S. R. (2013). Signs of adaptation to local $\mathrm{pH}$ conditions across an environmental mosaic in the California Current Ecosystem. Integr. Comp. Biol. 53, 857-870. doi: 10.1093/icb/ict094

Peter, I., and Davidson, E. H. (2010). Genomic programs for endoderm specification in sea urchin embryos. Dev. Biol. 344:469. doi: 10.1016/j.ydbio.2010.05.225

Peter, I. S., and Davidson, E. H. (2009). Modularity and design principles in the sea urchin embryo gene regulatory network. FEBS Lett. 583, 3948-3958. doi: 10.1016/j.febslet.2009.11.060

Peter, I. S., and Davidson, E. H. (2011). A gene regulatory network controlling the embryonic specification of endoderm. Nature 474, 635-639. doi: 10.1038 /nature10100

Petersen, C. P., and Reddien, P. W. (2009). Wnt signaling and the polarity of the primary body axis. Cell 139, 1056-1068. doi: 10.1016/j.cell.2009.11.035

Ransick, A., and Davidson, E. H. (2006). cis-regulatory processing of Notch signaling input to the sea urchin glial cells missing gene during mesoderm specification. Dev. Biol. 297, 587-602. doi: 10.1016/j.ydbio.2006.05.037

Ransick, A., and Davidson, E. H. (2012). Cis-regulatory logic driving glial cells missing: self-sustaining circuitry in later embryogenesis. Dev. Biol. 364, 259-267. doi: 10.1016/j.ydbio.2012.02.003

Runcie, D. E., Garfield, D. A., Babbitt, C. C., Wygoda, J. A., Mukherjee, S., and Wray, G. A. (2012). Genetics of gene expression responses to temperature stress in a sea urchin gene network. Mol. Ecol. 21, 4547-4562. doi: 10.1111/j.1365294x.2012.05717.x 
Saudemont, A., Haillot, E., Mekpoh, F., Bessodes, N., Quirin, M., Lapraz, F., et al. (2010). Ancestral regulatory circuits governing ectoderm patterning downstream of Nodal and BMP2/4 revealed by gene regulatory network analysis in an echinoderm. PLoS Genet. 6:e1001259. doi: 10.1371/journal.pgen.1001259

Shen-Orr, S. S., Milo, R., Mangan, S., and Alon, U. (2002). Network motifs in the transcriptional regulation network of Escherichia coli. Nat. Genet. 31, 64-68. doi: $10.1038 / \mathrm{ng} 881$

Shoval, O., and Alon, U. (2010). SnapShot: network motifs. Cell 143:326-e1. doi: 10.1016/j.cell.2010.09.050

Smith, J., Kraemer, E., Liu, H., Theodoris, C., and Davidson, E. (2008). A spatially dynamic cohort of regulatory genes in the endomesodermal gene network of the sea urchin embryo. Dev. Biol. 313, 863-875. doi: 10.1016/j.ydbio.2007.10.042
Smith, J., Theodoris, C., and Davidson, E. H. (2007). A gene regulatory network subcircuit drives a dynamic pattern of gene expression. Science 318, 794-797. doi: $10.1126 /$ science. 1146524

Conflict of Interest Statement: The author declares that the research was conducted in the absence of any commercial or financial relationships that could be construed as a potential conflict of interest.

Copyright (c) 2016 Ben-Tabou de-Leon. This is an open-access article distributed under the terms of the Creative Commons Attribution License (CC BY). The use, distribution or reproduction in other forums is permitted, provided the original author(s) or licensor are credited and that the original publication in this journal is cited, in accordance with accepted academic practice. No use, distribution or reproduction is permitted which does not comply with these terms. 